\title{
Reversible Focal Cerebral Cortical Lesions in a Patient with Heat Stroke
}

\author{
Atsuhito Fuse ${ }^{1}$, Kazuo Yamashiro ${ }^{1}$, Yutaka Oji ${ }^{1}$, Tsuyoshi Furuya ${ }^{1}$, \\ Kazuyuki Noda ${ }^{1}$, Nobutaka Hattori ${ }^{2}$ and Yasuyuki Okuma ${ }^{1}$
}

\begin{abstract}
We herein report the rare case of a 56-year-old man who suffered from heat stroke. Although he was in a coma with convulsions on arrival and developed multiorgan failure, he recovered after two weeks of successful treatments. Hyperintense signals on the right temporoparietooccipital cortex, which disappeared within one week, were demonstrated on diffusion-weighted magnetic resonance images. A diagnosis of transient cortical injury caused by heat stroke was suggested. Although the cerebellum is most susceptible to lesion formation, the mechanisms underlying heat stroke are multifactorial and may result in a variety of brain lesions.
\end{abstract}

Key words: heat stroke, cerebral cortex, magnetic resonance imaging, diffusion-weighted images

(Intern Med 52: 377-380, 2013)

(DOI: 10.2169/internalmedicine.52.7645)

\section{Introduction}

Exposure to high environmental temperatures or strenuous exercise can cause thermoregulatory failure. The most severe condition is heat stroke, which results in consciousness disturbance, convulsions and multiorgan failure. Heat stroke is often fatal, and those who do survive may sustain permanent neurologic damage (1). The brain is one of the organs that is most vulnerable to heat stroke, and brain lesions caused by heat stroke are bilateral and symmetrical in most cases (2-8). We herein report the first case of a patient with heat stroke presenting with focal reversible cortical lesions demonstrated on diffusion-weighted magnetic resonance imaging (MRI).

\section{Case Report}

A 56-year-old man felt general malaise and lost consciousness, while working as a mountain trail guide on a hot summer day. Three hours passed before emergency medical services rescued him. His axillary temperature was $39.4^{\circ} \mathrm{C}$. External cooling was promptly initiated, and he was transferred to our hospital. On arrival, his bladder temperature was $39.6^{\circ} \mathrm{C}$, his blood pressure was $99 / 60 \mathrm{mmHg}$ and his heart rate was 132 beats/min. No obvious trauma was found, including head injury. The Glasgow Coma Scale score was 8 (E3, V1, M4), and tonic-clonic seizures of the bilateral arms were observed. Brain computed tomography showed no abnormal findings. The patient was immediately intubated, treated with anticonvulsants and admitted to the intensive care unit. External cooling was continued with cooling blankets. The patient developed severe liver dysfunction, rhabdomyolysis and disseminated intravascular coagulation (DIC), which was treated with transfusion of fresh frozen plasma. Brain MRI was performed three days after admission. Diffusion-weighted images (DWI) demonstrated hyperintense signals on the right temporoparietooccipital cortex (Fig. 1a). Fluid-attenuated inversion recovery (FLAIR) images obtained at the same time showed swelling of the cortical ribbon and decreased subcortical white matter signal intensity of the right temporoparietooccipital lobes (Fig. 1b). Electroencephalography (EEG) findings obtained the same day showed spikes originating predominantly from the right temporoparietooccipital region with secondary generalization during clinical seizures (Fig. 2). The right-side dominance was most prominent in the temporal (T4) and occipital (O2) electrodes at the end of the seizure discharges. A cerebrospi-

${ }^{1}$ Department of Neurology, Juntendo University Shizuoka Hospital, Japan and ${ }^{2}$ Department of Neurology, Juntendo University School of Medicine, Japan

Received for publication March 2, 2012; Accepted for publication October 21, 2012

Correspondence to Dr. Kazuo Yamashiro, kazuo-y@juntendo.ac.jp 


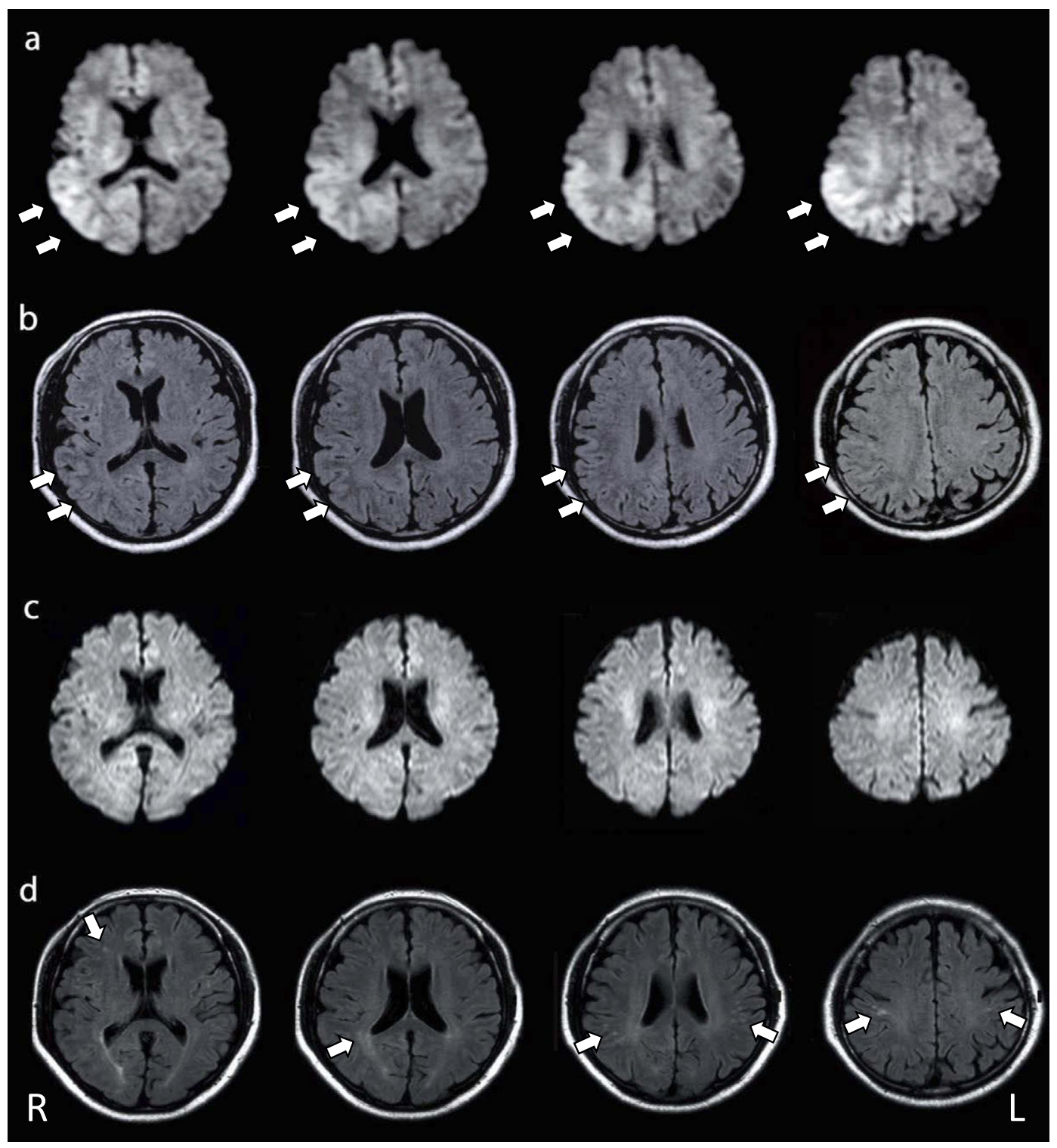

Figure 1. Brain MRI performed three days after admission (a, b). Diffusion-weighted images (DWI) demonstrated hyperintense signals on the right temporoparietooccipital cortex (arrows) (a). Fluid-attenuated inversion recovery (FLAIR) images showed swelling of the cortical ribbon and decreased subcortical white matter signal intensity (arrows) (b). Brain MRI performed six days after admission (c, d). DWI showed no abnormal signals (c). FLAIR images showed small hyperintense signals on the subcortical white matter (arrows) (d). (Toshiba EXCELART 1.5T. TR/TE=6,000/130 (a), 10,000/120 (b). Philips Intera 1.5T. TR/TE=2,932/100 (c), 6,000/150 (d). ) (R=right side, L=left side)

nal fluid (CSF) examination showed mild pleocytosis (white blood cells: 51 cells $/ \mathrm{mm}^{3}$; segmented neutrophils: 45 cells/ $\mathrm{mm}^{3}$; and lymphocytes: 6 cells $/ \mathrm{mm}^{3}$ ), a glucose level of 89 $\mathrm{mg} / \mathrm{dL}$ (serum glucose level: $146 \mathrm{mg} / \mathrm{dL}$ ) and a protein level of $56 \mathrm{mg} / \mathrm{dL}$. The patient's CSF pressure was elevated higher than $200 \mathrm{mmH}_{2} \mathrm{O}$. A CSF culture and PCR analysis of herpes simplex virus DNA showed negative findings. The patient's consciousness disturbance and seizures resolved rapidly and the laboratory abnormalities improved. Followup MRI performed six days after admission revealed hyperintense signals on the subcortical white matter on FLAIR images (Fig. 1d). EEG performed on day 13 showed no ab- normal findings. The patient was discharged from our hospital without abnormal neurological findings on day 15 .

\section{Discussion}

Heat stroke is characterized by elevated core temperatures higher than $40^{\circ} \mathrm{C}$ and central nervous system dysfunction that results in delirium, convulsions or coma (1). The temperature of our patient did not reach $40^{\circ} \mathrm{C}$ on admission. This may be due to the fact that he had already been externally cooled with cooling blankets in the ambulance. The clinical findings of consciousness disturbance, convulsions 
a

b

C

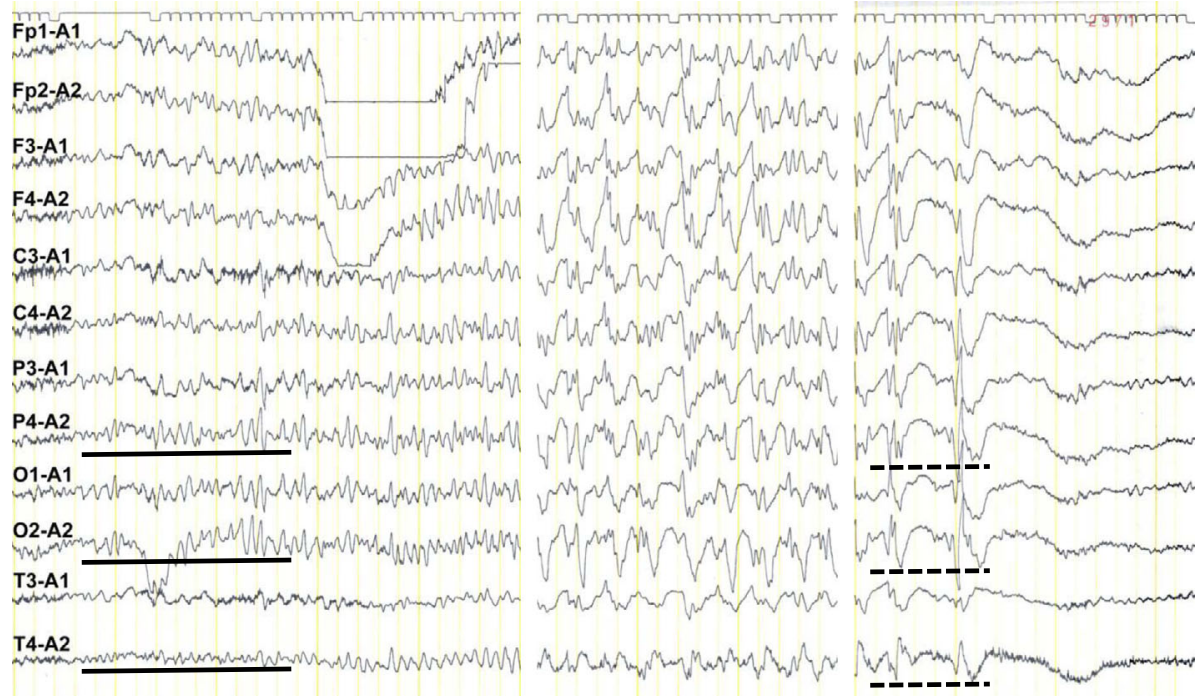

Figure 2. Electroencephalography (EEG) performed three days after admission. EEG performed at onset (a), 53 seconds after onset (b) and 85 seconds after onset (c) of a clinical seizure. Spikes appeared predominantly on the right temporoparietooccipital region (lines) (a) with secondary generalization (b). Laterality (right predominance) was most prominent at the end of the seizure discharges (dotted lines) (c).

and multiorgan dysfunction indicated that he had suffered from heat stroke.

To the best of our knowledge, this is the first report of a case of a heat stroke patient with focal reversible cortical injury demonstrated on MRI. EEG showed focal epileptic discharges from the lesion. The cerebellum is most susceptible to lesion formation in patients with heat stroke. Previous case studies using MRI have shown lesions or atrophy of the cerebellum as well as cerebellar symptoms such as limb ataxia (2-8). Such findings are explained by the selective vulnerability of Purkinje cells to heat-induced injury (3). However, lesions in the caudate nucleus (4), hippocampus $(4,5)$, external capsule $(6)$, thalamus $(6)$, splenium of the corpus callosum (7) and subcortical white matter (4) have also been detected on MRI. Cortical lesions have been observed in some heat stroke patients. Akaboshi et al. reported cortical laminar necrosis in the bilateral vascular boundary zones caused by decreases in blood flow in a 10month-old boy with heat stroke (9). However, vascular boundary zone abnormalities were not observed in our patient. Therefore, the mechanisms underlying the cortical injury in our patient may be different from those present in the patient reported by Akaboshi et al. Sudhakar et al. reported involvement of the cortex and cerebellum in a 2-yearold girl who suffered from heat stroke (5). Additionally, animal experiments have shown that rats exposed to heat stress frequently exhibit disruption of the blood-brain barrier in the cerebral cortices that results in vasogenic brain edema (10).

Damage to the brain caused by heat stroke may be multifactorial. Excessive production of cytokines can disrupt the blood-brain barrier, which results in vasogenic edema. Microthrombosis can result in small vessel ischemic damage, and heat itself can be directly cytotoxic. Brain damage can be transient or eventually result in irreversible injury $(1,4)$. Transient hyperintense signals in the right temporoparietooccipital cortex on DWI in our patient suggest the presence of transient cortical injury that may have been caused by vasogenic edema. Fujioka et al. reported the usefulness of the apparent diffusion coefficient (ADC) for differentiating vasogenic edema from cytotoxic edema in a heat stroke patient (8). Cytotoxic edema in the cerebellar cortex in their patient was suggested by the presence of hyperintense signals on DWI and a decrease in the ADC. Two months after the onset of heat stroke, dysmetria of the extremities in their patient worsened, and the cerebellum became atrophic. A diagnosis of irreversible neuronal damage caused by cytotoxic edema was considered. Because our patient did not develop any sequelae, follow-up MRI was not performed after discharge. However, follow-up MRI should be performed in patients with residual neurological findings. Subcortical white matter lesions compatible with small vessel disease were also detected on FLAIR images on follow-up MRI of our patient performed during hospitalization. These findings may have been associated with DIC, which was observed in our patient on admission. DIC can cause microthrombosis and results in small vessel ischemic damage $(1,4)$.

Another unique finding in our patient was focal lesions. Most previous case reports have reported bilateral lesions in heat stroke patients. However, even in one patient with bilateral cerebellar lesions, the size of the lesions differed between the hemispheres (4). Furthermore, the bilateral cortical lesions were not symmetric in one heat stroke patient (5). These findings suggest that the threshold of lesion formation for heat stroke may differ; alternatively, the sever- 
ity of heat stress cannot always be the same in both hemispheres. Although our patient showed focal lesions, the long duration of heat exposure might also have caused the bilateral lesions observed in this case.

It may be argued that the cortical lesions revealed on MRI in our patient were merely the consequence of convulsive seizures. MRI can show transient abnormal signals in the cortex during status epilepticus (11). It is very difficult to determine whether these findings reflect either the primary lesions or the post-epileptic changes only. We therefore reasonably assume that both the primary changes due to heat stroke and the secondary cortical changes due to seizures might have contributed to the MRI findings observed in this case.

In conclusion, physicians should recognize that the mechanisms underlying heat stroke are multifactorial and may result in a variety of brain lesions. Providing early diagnosis and management of heat stroke is very important because it may determine the prognosis.

The authors state that they have no Conflict of Interest (COI).

\section{References}

1. Bouchama A, Knochel JP. Heat stroke. N Engl J Med 346: 19781988, 2002.
2. Albukrek D, Bakon M, Moran DS, Faibel M, Epstein Y. Heatstroke-induced cerebellar atrophy: clinical course, CT and MRI findings. Neuroradiol 39: 195-197, 1997.

3. Bazille $C$, Megarbane $B$, Bensimhon $D$, et al. Brain damage after heat stroke. J Neuropathol Exp Neurol 64: 970-975, 2005.

4. Mahajan S, Schucany WG. Symmetric bilateral caudate, hippocampal, cerebellar, and subcortical white matter MRI abnormalities in an adult patient with heat stroke. Proc (Bayl Univ Med Cent) 21: 433-436, 2008.

5. Sudhakar PJ, Al-Hashimi H. Bilateral hippocampal hyperintensities: a new finding in MR imaging of heat stroke. Pediatr Radiol 37: 1289-1291, 2007.

6. McLaughlin CT, Kane AG, Auber AE. MR imaging of heat stroke: external capsule and thalamic T1 shortening and cerebellar injury. AJNR Am J Neuroradiol 24: 1372-1375, 2003.

7. Lee JS, Choi JC, Kang SY, Kang JH, Park JK. Heat stroke: increased signal intensity in the bilateral cerebellar dentate nuclei and splenium on diffusion-weighted MR imaging. AJNR Am J Neuroradiol 30: E58, 2009.

8. Fujioka Y, Yasui K, Hasegawa Y, Takahashi A, Sobue G. An acute heat stroke patient showing abnormal diffuse high intensity of the cerebellar cortex in diffusion weighted image: a case report. Clin Neurol 49: 634-640, 2009.

9. Akaboshi S, Miyashita A. A case of heat stroke with cortical laminar necrosis on vascular boundary zones. No to Hattatsu 28: 434437, 1996.

10. Sharma HS, Hoopes PJ. Hyperthermia induced pathophysiology of the central nervous system. Int J Hyperthermia 19: 325-354, 2003.

11. Lansberg MG, O'Brien MW, Norbash AM, Moseley ME, Morrell M, Albers GW. MRI abnormalities associated with partial status epilepticus. Neurology 52: 1021-1027, 1999.

(C) 2013 The Japanese Society of Internal Medicine http://www.naika.or.jp/imonline/index.html 